\title{
Occupational impact of internet-delivered cognitive behaviour therapy for depression and anxiety: reanalysis of data from five Australian randomised controlled trials
}

\section{Anna Mackenzie MPH \\ Samuel Harvey MRCPsych, FRANZCP, PhD \\ Senior Lecturer and \\ Research Fellow ${ }^{2}$ \\ Louise Mewton \\ BA(Hons), PhD \\ Gavin Andrews \\ MD, FRANZCP \\ Professor 1,3 \\ 1 Clinical Research Unit for Anxiety and Depression University of \\ New South Wales \\ Sydney, NSW. \\ 2 Black Dog Institute University of \\ New South Wales \\ Sydney, NSW. \\ 3 Anxiety and Depression Clinic, St Vincent's Hospital, Sydney, NSW. \\ louisem@unsw.edu.au}

MJA 2014; 201: 417-419 doi: 10.5694/mjal4.00293
T he Australian Government has invested about $\$ 180$ million into online mental health and telephone crisis support services, ${ }^{1}$ and Australian researchers are international leaders in the development of online mental health programs. ${ }^{2}$ Internet-delivered cognitive behaviour therapy (iCBT) courses are efficacious for anxiety and depressive disorders (the number needed to treat is about two); they significantly reduce symptoms and disability associated with these disorders. $^{2}$

Depression and anxiety are associated with high levels of occupational disability. ${ }^{3}$ They are the leading cause of sickness absence in most developed countries and account for about $35 \%$ of disability benefits. ${ }^{4}$ Left untreated, these disorders can lead to significant costs to employers, government, individuals and society. ${ }^{5}$

A few studies have shown that treatments for anxiety and depression improve occupational functioning. In depressed individuals, antidepressant medication has been shown to increase work productivity $^{6}$ and psychodynamic therapy (with or without adjunctive antidepressant medication) has been shown to reduce self-reported absenteeism. ${ }^{7,8}$ In individuals with anxiety disorders, face-to-face CBT has been shown to increase work productivity. ${ }^{9}$ For a range of anxiety and affective disorders, face-to-face CBT combined with occupational therapy has been shown to improve occupational functioning. ${ }^{10}$ Only one study, focusing on depression alone, has demonstrated increased productivity after iCBT. ${ }^{11}$

We examined data from five randomised controlled trials (RCTs) of anxiety and depression to assess whether completion of iCBT courses

\section{Abstract}

Objective: To determine whether internet-delivered cognitive behaviour therapy (iCBT) for depression and anxiety reduces self-reported absenteeism in employed individuals.

Design, participants and setting: We reanalysed data from five randomised controlled trials of iCBT: two for depression (conducted from September 2008 to February 2009 and from June 2009 to January 2010), two for generalised anxiety disorder (conducted from March 2009 to June 2009 and from July 2009 to January 2010) and one for social phobia (conducted from May 2008 to July 2008). Participants across Australia were recruited via a website. The inclusion criteria were: (i) meets criteria for the disorder of interest; (ii) aged 18 years or over; (iii) no previous history of a psychotic disorder or drug or alcohol misuse; (iv) not actively suicidal. The iCBT courses each consisted of six online lessons (to be completed within 11 weeks), homework assignments, automatic emails and resource documents.

Main outcome measure: The number of days absent (self-reported absenteeism) in the previous week.

Results: We included 284 participants in our analysis. When data for the three disorders were combined, participants who received iCBT had significant reductions in self-reported absenteeism compared with those in the control groups (who were on a waitlist) $(P=0.03)$. When data for the three disorders were analysed separately, reductions in self-reported absenteeism for participants who received iCBT were not significantly different to those for participants in the control groups.

Conclusion: Using data from five RCTs, we showed that iCBT was associated with reductions in self-reported absenteeism. Future research should focus on replicating these findings in other contexts, such as other disorders and other iCBT courses.

results in fewer work days being lost due to illness.

\section{Methods}

We used data from five RCTs conducted between 2008 and 2010 that established the efficacy of iCBT for depression (two trials, conducted from September 2008 to February 2009 and from June 2009 to January 2010), ${ }^{12,13}$ generalised anxiety disorder (two trials, conducted from March 2009 to June 2009 and from July 2009 to January 2010) $)^{14,15}$ and social phobia (one trial, conducted from May 2008 to July 2008). ${ }^{16}$ In each of these trials, participants were specifically asked about days absent from work (self-reported absenteeism). Data on reduced symptoms, presence of disorder and disability have previously been reported from these trials, but data on self-reported absenteeism have not. To ensure sufficient power to compare treatment effects across disorders, data from the two depression trials were combined, as were data from the two generalised anxiety disorder trials.

Participants: Recruitment of participants for the trials was done via a website (https://thiswayup. org.au). Participants completed questionnaires that included questions on severity and chronicity of symptoms and demographic details. Inclusion criteria were the same for the five trials: (i) meets Diagnostic and statistical manual of mental disorders (fourth edition) criteria for the disorder of interest, as determined by the Mini International Neuropsychiatric Interview Version 5.0.0;17 (ii) aged 18 years or over; (iii) no previous history of a psychotic disorder or drug 


\section{Participant flow diagram}

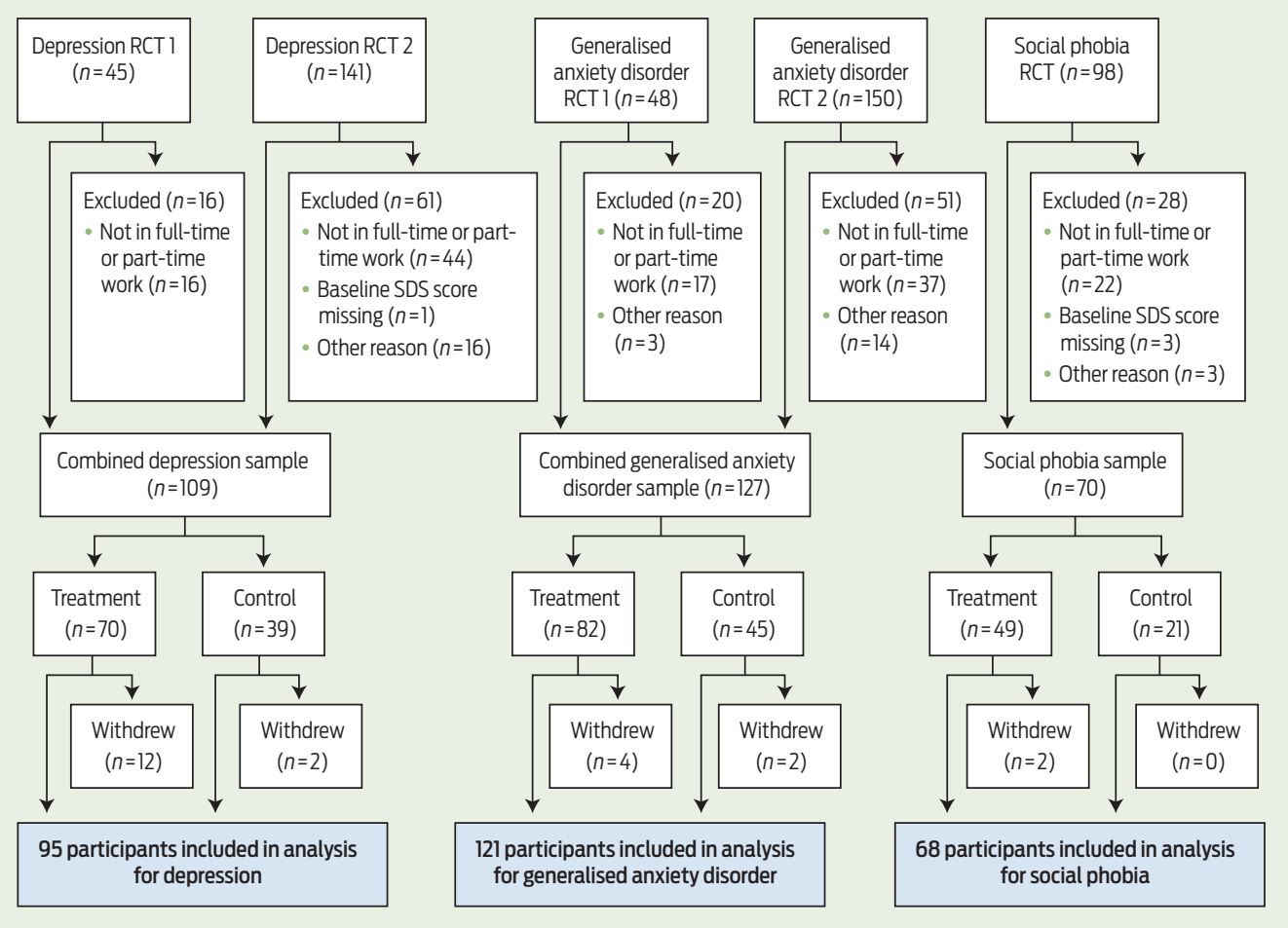

$\mathrm{RCT}=$ randomised controlled trial. SDS = Sheehan Disability Scale. on the REPEATED subcommand. Model fit indices and inspection of the variance-covariance matrix supported the specification of a diagonal covariance structure that assumes unequal variances and zero covariances. ${ }^{19}$ Analyses were conducted separately for the depression, generalised anxiety disorder and social phobia groups, as well as for the three groups combined. Effect sizes based on Cohen's $d$ were also calculated.

Ethics: All five trials were approved by the University of New South Wales Human Research Ethics Committee and registered with the Australian New Zealand Clinical Trials Registry.

\section{Results}

A total of 482 participants entered the five trials included in our study (Box 1). Mean age of the 306 participants who were eligible for inclusion in our study was 43 years (range, $18-68$ years), and 214 of them (70\%) were women. A total of 284 participants from this reduced sample (93\%) provided data on absenteeism after completing the relevant iCBT course or after the waitlist period.

When data for the three disorders were analysed separately, reductions in self-reported absenteeism for participants who received iCBT were not significantly different to those for participants in the control groups (Box 2). However, when data for the three disorders were combined, participants who received iCBT reported halving their work loss days $(P=0.03)$ (Box 2).

how many days in the last week did your symptoms cause you to miss work?"18 The questionnaire was administered immediately before treatment and 1 week after the final lesson of treatment.

Statistical analysis: Analyses were conducted using linear mixed-model repeated-measures analysis of variance with time (before $\mathrm{v}$ after) as a within-group factor and intervention (treatment $\mathrm{v}$ control) as a betweengroup factor. These analyses were conducted using the linear mixedeffects models (MIXED) procedure in SPSS, Version 19 (IBM), with a random effect for subject. This model was run with several different covariance structures specified

\section{Discussion}

Using data from five RCTs of iCBT for anxiety and depressive disorders, we showed that iCBT was associated with reductions in self-reported absenteeism. Before treatment, rates of self-reported absenteeism were high, especially compared with the Australian average of 8.75 days per year. ${ }^{18}$

Relatively little is known about which interventions affect occupational attendance. ${ }^{20}$ The large sample size of our study and the RCT methods represent a significant contribution to knowledge in the area of occupational psychiatry. 


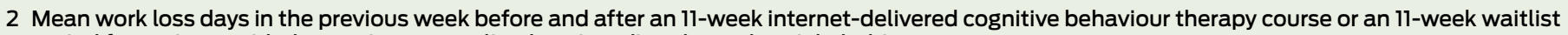
period for patients with depression, generalised anxiety disorder and social phobia

\begin{tabular}{|c|c|c|c|c|c|c|c|c|}
\hline & \multicolumn{2}{|c|}{ Depression $(n=109)$} & \multicolumn{2}{|c|}{$\begin{array}{l}\text { Generalised anxiety } \\
\text { disorder }(n=127)\end{array}$} & \multicolumn{2}{|c|}{ Social phobia $(n=70)$} & \multicolumn{2}{|c|}{ Total $(n=306)$} \\
\hline & Before & After & Before & After & Before & After & Before & After \\
\hline Estimated marginal mean (SE) for treatment group & $0.86(0.18)$ & $0.41(0.16)$ & $0.60(0.14)$ & $0.40(0.16)$ & $0.41(0.15)$ & $0.18(0.10)$ & $0.64(0.09)$ & $0.35(0.09)$ \\
\hline Estimated marginal mean (SE) for control group & $0.84(0.24)$ & $1.00(0.20)$ & $0.96(0.19)$ & $1.13(0.22)$ & $0.52(0.23)$ & $0.38(0.15)$ & $0.83(0.13)$ & $0.93(0.12)$ \\
\hline Effect size based on Cohen's $d^{*}$ & \multicolumn{2}{|c|}{0.34} & \multicolumn{2}{|c|}{0.29} & \multicolumn{2}{|c|}{0.10} & \multicolumn{2}{|c|}{0.32} \\
\hline$P$ for reduction in work loss days ${ }^{\dagger}$ & \multicolumn{2}{|c|}{0.10} & \multicolumn{2}{|c|}{0.15} & \multicolumn{2}{|c|}{0.72} & \multicolumn{2}{|c|}{0.03} \\
\hline
\end{tabular}

Our study has some limitations. The outcome measures relied on self-reported data; however, similar self-reported data have been shown to correspond closely with objective measures of work attendance. ${ }^{21,22}$ Limited demographic data were collected, and the effect of potentially important background and clinical characteristics (such as socioeconomic status, occupation type, and comorbid mental and physical conditions) could not be determined. Despite the use of appropriate analytic methods, missing post-treatment data, while minimal, may have biased the results in unknown ways. We only included iCBT courses from one clinic, and participants were recruited from a website (they may differ clinically from those receiving face-to-face treatment). Finally, use of a waitlist period as the control condition may have led to overestimation of treatment effects.

The importance of considering occupational outcomes of treatment interventions goes beyond economic arguments. Patients want to be fully functional in their occupation and many find that the benefits of work help in their recovery. A recent review concluded that there was insufficient evidence to show that standard interventions for depression (pharmacological and psychological) improve occupational health outcomes in depressed workers. ${ }^{23}$ Against this background, our findings that iCBT generates significant occupational benefits in addition to reducing symptoms are important. Future research should focus on replicating these findings in other contexts other disorders, other iCBT courses, and socioeconomic groups outside of Australia should be studied.

Acknowledgements: We thank the patients for completing the questionnaires and the research and clinical staff at the Clinical Research Unit for Anxiety and Depression who worked on the trials on which this study is based.

Competing interests: No relevant disclosures.

Received 3 Jan 2014, accepted 21 May 2014

1 Australian Government Department of Health and Ageing. E-mental health strategy for Australia. Canberra: DoHA, 2012. http://www. health.gov.au/internet/main/publishing.nsf/ Content/mental-pubs-e-emstrat (accessed May 2014).

2 Andrews G, Cuijpers P, Craske MG, et al. Computer therapy for the anxiety and depressive disorders is effective, acceptable and practical health care: a meta-analysis. PLOS One 2010; 5: el3196.

3 Harvey SB, Glozier N, Henderson M, et al. Depression and work performance: an ecological study using web-based screening. Occup Med (Lond) 2011; 61: 209-211.

4 Harvey SB, Henderson M, Lelliott P, Hotopf M. Mental health and employment: much work still to be done. Br J Psychiatry 2009; 194: 201-203.

5 Organisation for Economic Co-operation and Development. Sick on the job? Myths and realities about mental health and work. Paris: OECD Publishing, 2012.

6 Burnand Y, Andreoli A, Kolatte E, et al. Psychodynamic psychotherapy and clomipramine in the treatment of major depression. Psychiatr Serv 2002; 53: 585-590.

7 Knekt P, Lindfors O, Laaksonen MA, et al. Effectiveness of short-term and long-term psychotherapy on work ability and functional capacity - a randomized clinical trial on depressive and anxiety disorders. J Affect Disord 2008; 107: 95-106.

8 Rush AJ, Bose A. Escitalopram in clinical practice: results of an open-label trial in a naturalistic setting. Depress Anxiety 2005; 21: 26-32.

9 Linden M, Zubrägel D, Bär T. Occupational functioning, sickness absence and medication utilization before and after cognitive-behaviour therapy for generalized anxiety disorders. Clin Psychol Psychother 2011; 18: 218-224.

10 Rollman BL, Belnap BH, Mazumdar S, et al. A randomized trial to improve the quality of treatment for panic and generalized anxiety disorders in primary care. Arch Gen Psychiatry 2005; 62: 1332-1341.
11 Gerhards SA, de Graaf LE, Jacobs LE, et al. Economic evaluation of online computerised cognitive-behavioural therapy without support for depression in primary care: randomised trial. Br J Psychiatry 2010; 196: 310-318.

12 Perini S, Titov N, Andrews G. Clinician-assisted internet-based treatment is effective for depression: randomized controlled trial. Aust $N$ Z J Psychiatry 2009; 43: 571-578.

13 Titov N, Andrews G, Davies M, et al. Internet treatment for depression: a randomized controlled trial comparing clinician vs. technician assistance. PLOS One 2010; 5 : el0939.

14 Robinson E, Titov N, Andrews G, et al. Internet treatment for generalized anxiety disorder: $a$ randomized controlled trial comparing clinician vs. technician assistance. PLOS One 2010; 5: el0942.

15 Titov N, Andrews G, Robinson E, et al. Clinicianassisted internet-based treatment is effective for generalized anxiety disorder: randomized controlled trial. Aust N Z J Psychiatry 2009; 43: 905-912.

16 Titov N, Andrews G, Choi l, et al. Shyness 3: randomized controlled trial of guided versus unguided internet-based CBT for social phobia. Aust N Z J Psychiatry 2008; 42: 1030-1040.

17 Sheehan DV, Lecrubier Y, Sheehan KH, et al. The Mini-International Neuropsychiatric Interview (MINI): the development and validation of a structured diagnostic psychiatric interview for DSM-IV and ICD-10. J Clin Psychiatry 1998; 59 Suppl 20: 22-33.

18 Sheehan D, Harnett-Sheehan K, Raj B. The measurement of disability. Int Clin Psychopharmacol 1996; 11 Suppl 3: 89-95.

19 West BT, Welch KB, Galecki AT. Linear mixed models: a practical guide using statistical software. Boca Raton, Fla: Chapman and Hall/ CRC, 2006.

20 Henderson M, Harvey SB, Overland S, et al. Work and common psychiatric disorders. JR SOC Med 2011; 104: 198-207.

21 Revicki DA, Irwin D, Reblando J, Simon GE. The accuracy of self-reported disability days. Med Care 1994; 32: 401-404.

22 Ferrie JE, Kivimäki M, Head J, et al. A comparison of self-reported sickness absence with absences recorded in employers' registers: evidence from the Whitehall II study. Occup Environ Med 2005; 62: 74-79.

23 Nieuwenhuijsen K, Bültmann U, NeumeyerGromen A, et al. Interventions to improve occupational health in depressed people. Cochrane Database Syst Rev 2008; (2): CD006237. 